Instituto Internacional de Investigación y Desarrollo Tecnológico Educativo INDTEC, C.A.

DOI: https://doi.org/10.29394/Scientific.issn.2542-2987.2020.5.18.12.234-252

OAI-PMH: http://www.indteca.com/ojs/index.php/Revista Scientific/oai

Artículo Original / Original Article

\title{
Percepción del alumnado del grado de educación primaria sobre sostenibilidad y el urbanismo (Córdoba, España)
}

Autores: Miguel Jesús López Serrano

Universidad de Córdoba, UCO

milopez@uco.es

Córdoba, España

https://orcid.org/0000-0002-3953-1998

Rafael Guerrero Elecalde

Universidad de Córdoba, UCO

rgelecalde@uco.es

Córdoba, España

https://orcid.org/0000-0003-4271-897X

\section{Resumen}

Actualmente, uno de los desafíos educativos fundamentales en la formación docente es trabajar los Objetivos de Desarrollo Sostenible establecidos en la Agenda 2030, en la Cumbre para el Desarrollo Sostenible, realizada en la Sede de las Naciones Unidas (2015). En este sentido, el objetivo del artículo es analizar la sostenibilidad en la ciudad de Córdoba (España), por un lado, teniendo en cuenta el desarrollo urbano de las últimas décadas y, por otro, la percepción de los estudiantes de Bachillerato de Educación Primaria sobre esta ciudad como una comunidad sostenible, por medio de una metodología activa y participativa situando al alumnado en el centro del proceso de enseñanza-aprendizaje con el objetivo de conseguir un aprendizaje significativo a la par que lúdico. Posteriormente a la actividad, se preguntó al alumnado, a través de un formulario, para reflexionar sobre los contenidos y el diseño de la docencia de las Ciencias Sociales.

Palabras clave: sostenibilidad; medioambiente; didáctica de las ciencias sociales.

Cómo citar este artículo:

López, M., \& Guerrero, R. (2020). Percepción del alumnado del grado de educación primaria sobre sostenibilidad y el urbanismo (Córdoba, España). Revista Scientific, 5(18), 234-252, eISSN: 2542-2987. Recuperado de: https://doi.org/10.29394/Scientific.issn.25422987.2020.5.18.12.234-252

Fecha de Recepción: 30-06-2020
Fecha de Aceptación: 11-09-2020
Fecha de Publicación: 05-11-2020 
OAI-PMH: http://www.indteca.com/ojs/index.php/Revista_Scientific/oai

Artículo Original / Original Article

\title{
Perception of the students of the degree of primary education on sustainability and urban planning (Córdoba, Spain)
}

\begin{abstract}
Currently, one of the fundamental educational challenges in teacher training is to work on the Sustainable Development Goals established in the 2030 Agenda, at the Summit for Sustainable Development, held at the Headquarters United Nations (2015). In this sense, the objective of the article is to analyze sustainability in the city of Córdoba (Spain), on the one hand, taking into account the urban development of the last decades and, on the other, the perception of the students of the Baccalaureate of Primary Education about this city as a sustainable community, through an active and participatory methodology, placing students at the center of the teaching-learning process with the aim of achieving meaningful and playful learning. After the activity, the students were asked, through a form, to reflect on the contents and design of the teaching of Social Sciences.
\end{abstract}

Keywords: sustainability; environment; teaching social sciences.

\section{How to cite this article:}

López, M., \& Guerrero, R. (2020). Perception of the students of the degree of primary education on sustainability and urban planning (Córdoba, Spain). Revista Scientific, 5(18), 234-252, e-ISSN: 2542-2987. Recovered from: https://doi.org/10.29394/Scientific.issn.2542-2987.2020.5.18.12.234$\underline{252}$

Date Received: 30-06-2020
Date Acceptance: 11-09-2020
Date Publication: 05-11-2020 


\section{Introducción}

Uno de los desafíos más concluyentes que tiene planteado en la actualidad el crecimiento y la planificación de las ciudades, es una expansión territorial y urbana sostenible. Lo que implicaría, el contribuir a expandir el progreso en base a las obligaciones que requiere hacer efectiva la sostenibilidad y a los condicionantes económicos, sociales, ambientales y educativos.

Desde hace más de una década, la colaboración entre los diferentes gobiernos europeos y mundiales ha girado en torno a la sostenibilidad de las urbes. En este sentido, se aprobó la Carta de Leipzig (2007): sobre Ciudades Europeas Sostenibles, resultado del compromiso adquirido por los países miembros para elaborar un marco de referencia que aglutinara las políticas de desarrollo urbano y comenzar un debate sobre la integración de los planteamientos para la sostenibilidad de las ciudades atendiendo a los diferentes niveles: nacionales, regionales y locales de los estados comunitarios siguiendo la filosofía de la Declaración de Bristol del 6 de diciembre del año 2005. En los acuerdos de Leipzig, se estipularon una serie de recomendaciones y estrategias de acción, destacando: Modernizar las redes de infraestructuras y mejora de la eficiencia energética, realizar una innovación proactiva y políticas educativas, buscar estrategias para la mejora del medio ambiente físico, fomentar un transporte urbano eficiente y asequible...

Al año siguiente, en el 2008, bajo la presidencia europea de Francia se impulsó el Marco Europeo de Referencia para la Ciudad Sostenible (RFSC); una iniciativa web creada, cuya decisión de llevarla a efecto no era vinculante, para promover la sostenibilidad en las ciudades europeas, desarrollando logística y operativamente los principios recogidos en la "Carta de Leipzig". La importancia de este proyecto radica en el acuerdo de utilizar unos criterios comunes y una misma estructura para significar la sostenibilidad en las 
ciudades del viejo continente siendo capaz de adaptarse a las pluralidades sociales, políticas, culturales dentro del panorama europeo. Desde el año 2017, la herramienta se ha enriquecido para integrar otros marcos en favor del desarrollo urbano sostenible. En términos más generales, SánchezHernández y Maldonado-Briegas (2019): harán un seguimiento de las diversas propuestas que contribuyen a la ampliación e inclusión de las ciudades sostenibles en toda Europa.

La sostenibilidad urbana, como afirman McMichael, Butler y Folke (2003): es un proceso que se prevé largo (somos conscientes) y que demanda a las administraciones públicas, a través de una sensibilización colectiva, una serie de medidas y acciones concretas. En el caso concreto de España, el gobierno aprobó en el año 2007 un plan de acción que acometiera los dictámenes de la Estrategia Europea de Desarrollo Sostenible (EUSDS). En este sentido, Mogren y Gericke (2017): entienden que la Estrategia Española de Desarrollo Sostenible (EEDS) se articula en torno a 3 áreas: la sostenibilidad ambiental, social y global.

En relación a la sostenibilidad del entorno urbano que nos ocupa en estas líneas, la EEDS, desarrolla una serie de dimensiones para tratar de alcanzar un equilibrio factual entre urbanismo y medio ambiente en base a una planificación equilibrada del desarrollo territorial urbano y rural, prestando especial hincapié en los usos del suelo y la ocupación del territorio, estructurando los hábitats residenciales existentes y futuros mediante un proyecto de ciudad basado en una reformulación de los actividades económicas, ambientales y sociales, a través de una participación ciudadana activa.

A nivel local, en la ciudad de Córdoba, España, paralelamente TorresPorras y Arrebola (2018): destacan especialmente la ausencia de una oficina municipal dedicada íntegramente al Medio Ambiente, liderada por un profesional público altamente capacitado, con un presupuesto lo 
suficientemente amplio como para poder llevar a cabo los diversos proyectos o iniciativas que, a día de hoy se llevan a cabo desde el ayuntamiento con un margen de mejora amplio. Creemos por tanto que este elemento debe ser una prioridad para la corporación que gobierne la ciudad la siguiente legislatura.

Según González (2017): la construcción de instrumentos que optimicen la sostenibilidad de los diferentes sectores municipales debe ser otra de las prioridades que las corporaciones municipales deben de recoger en su proyecto de gobernabilidad, más aún, ante la situación de emergencia ambiental, económica, sanitaria y energética en la que nos encontramos en la actualidad. Asimismo, Zhai, Sun, Tsai, Wang, Zhao y Chen (2018): aseguran que la urbe tiene que mejorar y reorganizar todas sus herramientas para conseguir a corto o medio plazo, un sistema energético de bajo consumo en el que se sustituya de manera integral el impacto de los combustibles fósiles, entre otras cuestiones relacionadas con la sostenibilidad urbana. Aunque las competencias municipales en gestión de residuos son limitadas, el ayuntamiento debe desempeñar un papel destacado a la hora de reivindicar, a las administraciones competentes, la implantación de medidas, como la del propio Sistema de Depósito, Devolución y Retorno (SDDR), permitan un avance decidido hacia el Residuo Cero.

Por otro lado, en estos últimos años no se ha producido un crecimiento de nuevas zonas verdes al ritmo que necesita la ciudad de Córdoba, especialmente son necesarios amplios parques que complete el anillo verde tal como está contemplado en el Plan General de Ordenación Urbana.

La calidad del aire es otra de las asignaturas pendientes en nuestra ciudad. Desde hace años en varias ocasiones se han superado los límites de $\mathrm{NO}_{2}, \mathrm{SO}_{2}$, Ozono e incluso Cadmio. Con la subida de las temperaturas, causadas sobre todo como consecuencia del cambio climático, los efectos negativos de los contaminantes se potencian, por tanto, en Andalucía y especialmente en el Valle del Guadalquivir, debemos ser más exigentes con 
el control de la contaminación. En concreto, siguiendo a Giddens (2010): vivimos en una de las zonas de España con mayor índice de contaminación por Ozono troposférico.

En este sentido, el ritmo de incremento de la temperatura crecerá en los próximos años pudiendo suponer para finales de este siglo un aumento de la media anual en Córdoba de $3,5{ }^{\circ} \mathrm{C}$ acorde con los datos de las Naciones Unidas, proyectados a la comunidad autónoma por la Junta de Andalucía, lo que supondría un incremento de la temperatura media en verano en nuestra ciudad de entre 5 y $7^{\circ} \mathrm{C}$, si estos datos numéricos se cumplen, nos dirigimos a veranos propios de un desierto inhabitable.

Las medidas de adaptación deben buscar que la ciudad genere un microclima incrementando exponencialmente las superficies arboladas, incrementando las zonas verdes, sustituyendo superficies pavimentadas por superficies permeables, fomentando la construcción y rehabilitación bioclimática de viviendas, equipando las calles y plazas con criterios climáticos, preparando equipamientos públicos que permitan albergar a la población vulnerable durante los días con mayores temperaturas, algo así como centros de estancia ciudadana durante las olas de calor.

Por todo, refrendando las palabras de Casey y Asamoah (2016): es de vital importancia la incorporación en el ámbito educativo de un lenguaje específico que normalice conceptos clave tales como la sostenibilidad, comercio justo, consumo responsable, políticas energéticas respetuosas con el medioambiente... La Ley Orgánica para la Mejora de la Calidad Educativa (LOMCE, 2013): recoge en varios apartados de su articulado conceptos como la adquisición de una conciencia en la conservación de recursos naturales, solidaridad intergeneracional y global; en las diversas etapas de la educación española.

La experiencia didáctica que aquí se presenta está estructurada en base a una pregunta clave: ¿Qué percepción tiene el alumnado universitario 
en formación de la sostenibilidad, los problemas medioambientales, etc.?. Para responder a la misma se ha tomado como ámbito de referencia la ciudad de Córdoba, España y como marco educativo los grados en educación primaria.

Esta pregunta general se ha consignado con otros varios interrogantes: ¿La educación medioambiental que recibo en el centro educativo me permite distinguir lo que es bueno de lo que es malo para la conservación del medio ambiente?; ¿Qué metodología es la más adecuada para trabajarlos en clase?.

Por tanto, la cuestión que plantea un interrogante de cariz general, así como las más específicas pueden dirigir de una manera u otra los objetivos y planteamientos que se pretender analizar y llevar a cabo. Igualmente, pretendemos completar este objetivo general con otros más específicos:

- Sensibilizar de la importancia que tiene la sostenibilidad y la educación ambiental en todos los niveles.

- Acercar al alumnado con su entorno más próximo para fomentar el conocimiento del medio natural, cultural y social a través del análisis de la problemática sostenible y medioambiental.

A partir de las preguntas que estructuran la investigación, contemplamos una serie de supuestos resultados. Con el objetivo de fomentar un aprendizaje significativo, se diseñaron una serie de actividades para trabajar, mediante una metodología constructivista, el concepto de sostenibilidad a través del ámbito local. Se empezó revisando el Decreto 97/2015, de 3 de marzo según el Boletín Oficial de la Junta de Andalucía (BOJA, 2015): por el que se establece la ordenación y las enseñanzas correspondientes a la Educación Primaria en Andalucía, haciendo especial hincapié en los contenidos del área de Ciencias Sociales y del área de la Naturaleza. 


\section{Metodología (Materiales y métodos)}

La experiencia que aquí planteamos se llevó a cabo con dos grupos de unos 115 alumnos de tercer curso del Grado de Educación Primaria de la asignatura de Didáctica de las Ciencias Sociales y Didáctica de las Ciencias Experimentales, de la Facultad de Ciencias de la Educación de la Universidad de Córdoba durante los cursos académicos 2019-2020.

Para determinar el alcance de los objetivos del proyecto y, por medio de una metodología activa y participativa situando al alumnado en el centro del proceso de enseñanza-aprendizaje, se tuvo como objetivo conseguir un aprendizaje significativo a la par que lúdico. Posteriormente a la actividad, se preguntó al alumnado, a través de un formulario de Google Forms, con el fin de que la actividad planteada supusiera un ejercicio de reflexión sobre los contenidos y el diseño de la docencia de las Ciencias Sociales; más concretamente, con los conceptos relacionados con el medioambiente y la sostenibilidad trabajados en el aula.

Respecto a la secuencia de actividades, los establecieron de este modo:

1. Análisis del Decreto $97 / 2015$, de 3 de marzo.

2. Lecturas complementarias de artículos, webs y monografías.

3. Debate sobre la información recopilada.

4. Realización de un breve dossier informativo.

5. Cumplimentación de un cuestionario sobre la tarea realizada.

Se inició la actividad organizando al alumnado en grupos compuestos por entre 4-6 integrantes para analizar la información del marco europeo para la ciudad sostenible que creían más relevante, así como, la estrategia española de sostenibilidad urbana y local del año 2011, con la temática de la actividad propuesta y una selección de artículos, webs... que complementara la información ofrecida. 
A continuación, se debatió en el aula la importancia de los principales cambios urbanísticos, medio ambientales... acaecidos en la ciudad de Córdoba; aclarándose que es una intervención diferente a las que habitualmente se realizan en el aula y que conlleva una preparación previa, tanto en el desarrollo de la misma como tras su realización. Una vez familiarizados con el significado pedagógico que conlleva nuestra esta actividad, eje fundamental de la actividad, se llevó a cabo una sesión práctica en el aula de nuevas tecnologías en la que, nuestro alumnado, tenía que cumplimentar un pequeño cuestionario de evaluación diagnóstica (elaborado con la aplicación de administración de encuestas Google Forms), que nos serviría de guía para efectuar de manera rápida y fácil una evaluación inicial sobre el grado de percepción y conocimiento, que los y las estudiantes poseían de cuestiones relativas al entendimiento del concepto de sostenibilidad y la posible implementación de políticas sostenibles en la ciudad de Córdoba.

Con respecto a la secuencia de actividades, referida en líneas anteriores, en el ejercicio número 4 , se organizó la confección de un pequeño dossier informativo, una vez realizada una búsqueda bibliográfica e informativa y debatido en clase. El alumnado, dividido en grupos, procedieron a seleccionar la información sobre la sostenibilidad en la ciudad de Córdoba sin atender a ninguna directriz por parte del profesorado; así pues, la confección de dicha herramienta pedagógica fue fruto de los intereses, gustos 0 motivaciones personales de los y las estudiantes.

Por último, se les pidió que de forma individualizada cumplimentaran un cuestionario de evaluación final sobre la temática propuesta configurado en torno a 10 preguntas, con 5 niveles, teniendo en cuenta que un valor de 1 significaba poco relevante y un valor de 5 , muy relevante; que nos permitieran, en primer lugar, medir sus actitudes y conocer el grado de conformidad del alumnado. Igualmente, se pretendía evaluar el proceso de enseñanza aprendizaje del alumnado de la asignatura de Didáctica de las Ciencias 
Sociales. Para la realización de los análisis estadísticos y las gráficas, se ha utilizado el programa estadístico informático IBM SPSS Statistics versión 26.

\section{Resultados (análisis e interpretación de los resultados)}

Como hacíamos referencia anteriormente, conocer el grado de percepción y conocimientos de nuestro alumnado supone un recurso que mejora enormemente el proceso de enseñanza aprendizaje de las Ciencias Sociales en educación primaria debido a los múltiples beneficios que ofrece. Por otro lado, el cuestionario, es una herramienta pedagógica que atrae la atención y estimula la comprensión y reflexión de los conceptos investigados, por medio de una alta motivación que propicia que se fomente su creatividad y pensamiento crítico y social.

Las variables de identificación en este caso relativa al género, nos indican que predomina el sexo femenino, con 55 mujeres 66,3\%, frente al masculino, con 28 varones, lo que supone un $33,7 \%$ del total de los participantes.

Con respecto a la edad, comprobamos en las respuestas que la mayoría se encuentran entre los 20 y 23 años 81,3\%; siendo minoritaria la representación de estudiantes mayores o con menores, en este intervalo de edad.

Una de las preguntas formuladas en el cuestionario, es la concerniente a la idea que tiene nuestro alumnado sobre si la formación medioambiental recibida en el grado les ayuda a discernir las actuaciones positivas o negativas para la conservación del medio ambiente. Se trataba de indagar el nivel de conocimientos previos de los estudiantes implicados en esta actividad. De las 83 respuestas, destaca sobremanera, que el $71.1 \%$ está, de acuerdo y muy de acuerdo en que la formación recibida es de calidad, mientras que el $24.1 \%$ se muestran poco convencidos o convencidas de ello.

Destacamos que, a lo largo de los cuatro años del grado de Educación 
Primaria de la Universidad de Córdoba, existe una asignatura específica que verse sobre la temática expuesta: Didáctica del Medio Ambiente en Educación Primaria, por lo que, consideramos que las respuestas no se fundamentan en base a las asignaturas optativas cursadas por el alumnado.

Con la intención de incidir más en el tratamiento didáctico y en el modo en el que los planes de estudios abordan la sostenibilidad de las ciudades, realizamos sendas preguntas para calibrar la percepción que sobre la cuestión tienen los futuros docentes.

Gráfico 1. ¿Puedo influir y contribuir a la conservación del medio ambiente con mis acciones?.

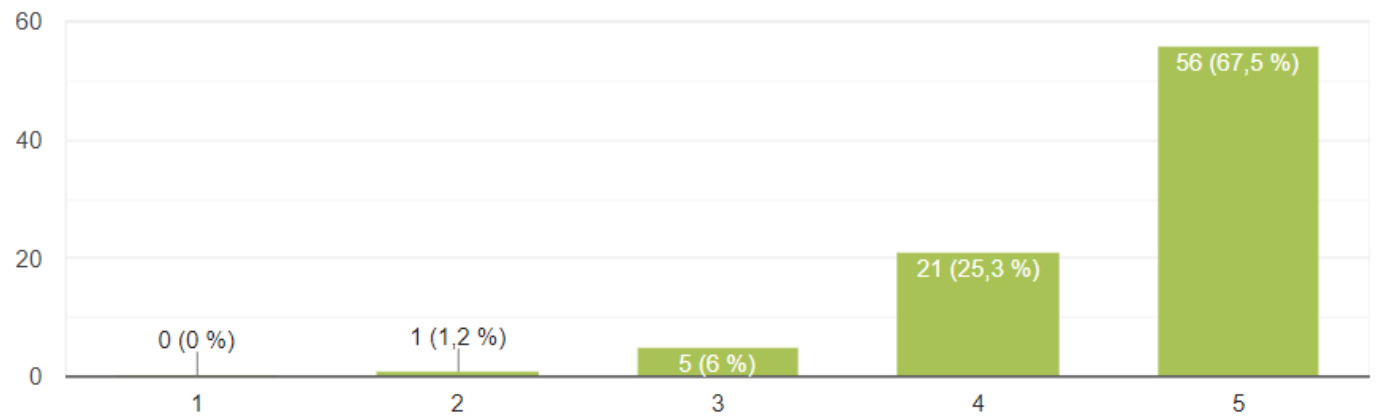

Fuente: Los Autores (2020).

El gráfico 1, recoge las contestaciones a la pregunta: ¿Puedo influir y contribuir a la conservación del medio ambiente con mis acciones? El 67.5\% (56 estudiantes) están de acuerdo en que la cotidianeidad de nuestras acciones puede influir tanto positiva como negativamente en la conservación del medio ambiente, el $25.3 \%$ se mostraban favorables a que nuestra conducta y modo de vida tiene incidencia en la sostenibilidad de nuestro hábitat local. Solo una persona, 1.2\% declaró no estar de acuerdo con la cuestión, mientras que el $6 \%$ de los alumnos y alumnas encuestados y encuestadas, se posicionaron de manera neutral. 
Gráfico 2. ¿La protección del medio ambiente implica la aplicación del concepto de desarrollo sostenible?.

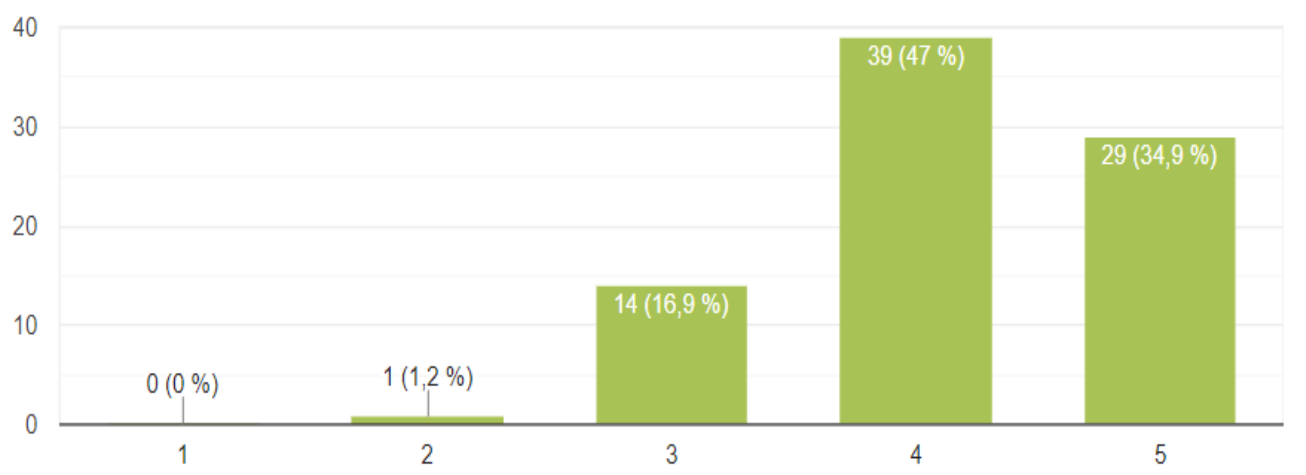

Fuente: Los Autores (2020).

Así mismo, las puntuaciones referidas a la cuestión: ¿La protección del medio ambiente implica la aplicación del concepto de desarrollo sostenible?, que se les planteaban en relación a si la protección del medio ambiente pasa por la aplicación del concepto del desarrollo sostenible, arrojan unos porcentajes claramente favorables, como se visualiza en el gráfico 2. En el caso, la inmensa mayoría de los y las estudiantes afirmaron estar de acuerdo, $47 \%$ o muy de acuerdo, $34,9 \%$ en que se puede actuar de una manera responsable teniendo en cuenta, a la hora de plantearnos una decisión, una serie de planteamientos referidos a la sostenibilidad, economía justa y solidaria y la igualdad social; elementos, como ya conocemos inherentes a las dimensiones que integran la sostenibilidad.

En que la metodología empleada en la actividad fomenta el trabajar contenidos de forma transversal. Por la tanto deducimos que, salvando grandes cuestiones, el alumnado detecta que urge realizar un cambio de actitud y, lo más importante, que para lograrlo hay que ser conscientes de que existe esta necesidad. Hay que reflexionar sobre las consecuencias que están teniendo y tendrán nuestras conductas como individuos en la conservación del 
medio ambiente. Igualmente, este tipo de actividades pueden funcionar como elementos capaces de vertebrar la comprensión de las identidades, el conocimiento de técnicas y recursos, y como fomentador de actitudes, dentro de la transversalidad pedagógica.

Igualmente, en respuesta a la pregunta sobre si nuestras acciones cotidianas no repercuten en la reducción del volumen de basura producido en la ciudad, 47 estudiantes $56.6 \%$ se mostraron a muy en desacuerdo de dicha afirmación. Hoy día se hace del todo innegable que consecuencia directa del estilo que se nos ha impuesto desde las grandes empresas, cada vez se nos hace más complicados discernir el nivel de impacto sobre el medioambiente de nuestras acciones cotidianas. El capitalismo imperante que marca nuestro actual ritmo de vida choca de pleno en la adquisición de unas políticas que generen un descenso considerable de los productos desechables, de las toneladas de plásticos y envases, los altísimos niveles de residuos generados..., acciones que no permiten llevar a la práctica una vida basada en las diferentes dimensiones de la sostenibilidad.

De igual manera, solo un $6 \%$ del alumnado encuestado no contempla la importancia que tiene una adquisición de hábitos de comportamiento sostenibles para una transformación social real. Mientras el $36.2 \%$ se muestran de acuerdo a los conceptos expresados en el proceso de Marrakech, consistente en dar solución a las necesidades vitales, sin implicar un mal uso de los recursos naturales y sin poner en peligro las necesidades de las generaciones venideras.

\section{Conclusiones}

Por los resultados recogidos podemos afirmar que el alumnado de la asignatura de Didáctica de las Ciencias Sociales del Grado de Primaria de la Universidad de Córdoba ha aceptado positivamente la práctica docente realizada para conocer el grado de percepción que tenían sobre la 
sostenibilidad de su ciudad. A través de la misma, se han convencido de que los problemas que afectan a la sostenibilidad no son parte exclusiva de las grandes empresas, ni de los políticos o gobiernos de turno, sino que, de una forma u otra, todos contribuimos con nuestro compromiso. Igualmente, en su vertiente profesional, los futuros docentes han entendido que este tipo de actuaciones educativas son un valioso recurso didáctico que pueden utilizar en el futuro, cuando se desempeñen como profesores y profesoras. Incluso algunos alumnos y alumnas habían recibido con anterioridad formación sobre la temática abordada y no habían llegado a estas conclusiones.

En este sentido, también comprenden, como bien lo expresan en el cuestionario, que para realizar cualquier actividad que tenga como fundamentación la sostenibilidad de las ciudades, el medio ambiente, la contaminación, etc., que el docente prepare y elabore a priori, y también con el alumnado, un trabajo de preparación, motivación y búsqueda de información, que ayude a extraer el máximo provecho de la experiencia didáctica.

Más allá de la estudio y enseñanza de la sostenibilidad en el ámbito académico, de gran valor para la asignatura Didáctica de las Ciencias Sociales, el alumnado ha aprendido sobre la realidad actual medio ambiental de la ciudad de Córdoba a través de la actividad que se les ha planteado y, especialmente, durante la realización de los diferentes ejercicios. Acorde a sus respuestas, lo han conseguido de forma amena, entretenida y diferente siendo posible gracias a las características temáticas abordadas.

Este artículo muestra que la experiencia pedagógica llevada a cabo por los estudiantes, aún debe de profundizar en muchos aspectos para que podamos hablar de que hay un conocimiento efectivo y práctico de lo que significa la sostenibilidad, a día de hoy en la realidad educativa de la mayoría de los centros escolares de la ciudad de Córdoba. Comprobándose que los principales argumentos esgrimidos en estas líneas hayan sido: la falta de una 
formación específica en sostenibilidad a lo largo del grado, una baja motivación a la hora de acometer el estudio de disciplinas que no albergan el peso específico dentro de los planes de estudio y el desconocimiento del marco institucional y legislativo no solo a nivel europeo o nacional sino, igualmente, a nivel local. No obstante, es un elemento ilusionante el que los futuros profesores encaren con motivación las metodologías y objetivos presentados dentro del marco medioambiental de la ciudad de la mezquita.

Se hace del todo necesario intervenir para llevar a efecto el cambio de esta situación, ya que son muchas y variadas las opciones pedagógicas para que cualquier centro educativo normalice la percepción sobre la sostenibilidad local, como una opción factible para la enseñanza-aprendizaje. Este cambio puede facultarse desde la universidad, en los grados de Educación, tal y como se muestra en este trabajo. Fomentando el conocimiento de las metodologías necesarias para organizar el conocimiento ecológico y sostenible, incitando la motivación del alumnado universitario de los grados de educación a participar en la metodología y la planificación de este recurso. Así, creemos que nuestro alumnado se familiarice, y pueda poner en práctica en un futuro cuando ejerzan profesionalmente en sus centros educativos unas prácticas educativas que prioricen el conocimiento de nuestra ciudad desde los parámetros marcados en la Cumbre para el Desarrollo Sostenible de las Naciones Unidas para la Agenda 2030 y los diversos objetivos de desarrollo sostenibles, para que pase, a ser una realidad educativa en las escuelas.

Por último, indicar que creíamos un objetivo fundamental a la hora de plantear un ejercicio de estas características, el inculcar a los docentes de nuestro futuro más cercano, que en última instancia deben de entender que la sostenibilidad de las ciudades son una parte fundamental tanto para la consecución de un aprendizaje significativo como para su formación personal y didáctica. 
Instituto Internacional de Investigación y Desarrollo Tecnológico Educativo INDTEC, C.A.

DOI: https://doi.org/10.29394/Scientific.issn.2542-2987.2020.5.18.12.234-252

OAI-PMH: http://www.indteca.com/ojs/index.php/Revista_Scientific/oai

Artículo Original / Original Article

\section{Referencias}

BOJA (2015). Decreto 97/2015, de 3 de marzo, por el que se establece la ordenación y el currículo de la Educación Primaria en la Comunidad Autónoma de Andalucía. Boletín Oficial de la Junta de Andalucía, (50), 1-436, ISSN: 2253 - 802X. Recuperado de:

https://www.juntadeandalucia.es/boja/2015/50/1

Carta de Leipzig (2007). Sobre Ciudades Europeas Sostenibles. Ciudad y territorio: Estudios territoriales, (152-153), 571-579, ISSN: 1133-4762. España: Dirección General de Arquitectura, Vivienda y Suelo del Ministerio de Fomento.

Casey, C., \& Asamoah, L. (2016). Education and sustainability: reinvigorating adult education's role in transformation, justice and development. International Journal of Lifelong Education, 35(6), 590606, e-ISSN: 0260-1370. Recovered from:

https://doi.org/10.1080/02601370.2016.1217281

Giddens, A. (2010). La política del cambio climático. Madrid, España: Alianza Editorial.

González, M. (2017). Estrategias Didácticas para el Fortalecimiento de los Valores Ambientalistas. Revista Scientific, 2(Ed. Esp.), 336-354, eISSN: 2542-2987. Recuperado de:

https://doi.org/10.29394/scientific.issn.2542-2987.2017.0.0.18.336-354 LOMCE (2013). Ley Orgánica 8/2013, de 9 de diciembre, para la mejora de la calidad educativa. BOE-A-2013-12886, Boletín Oficial del Estado. Madrid, España: Legislación Consolidada. Recuperado de:

https://www.boe.es/buscar/pdf/2013/BOE-A-2013-12886consolidado.pdf

McMichael, A., Butler, C., \& Folke, C. (2003). New Visions for Addressing Sustainability. Science, 302(5652), 1919-1920, e-ISSN: 1095-9203. Recovered from: https://doi.org/10.1126/science.1090001 
Instituto Internacional de Investigación y Desarrollo Tecnológico Educativo INDTEC, C.A.

DOI: https://doi.org/10.29394/Scientific.issn.2542-2987.2020.5.18.12.234-252

OAI-PMH: http://www.indteca.com/ojs/index.php/Revista_Scientific/oai

Artículo Original / Original Article

Mogren, A., \& Gericke, N. (2017). ESD implementation at the school organisation level, part 2 - investigating the transformative perspective in school leaders' quality strategies at ESD schools. Environmental Education Research, 23(7), 993-1014, e-ISSN: 13504622. Recovered from:

https://doi.org/10.1080/13504622.2016.1226266

Naciones Unidas (2015). Transformar nuestro mundo: la Agenda 2030 para el Desarrollo Sostenible. A/RES/70/1. Nueva York, Estados Unidos: La Asamblea General.

Sánchez-Hernández, M., \& Maldonado-Briegas, J. (2019). Sustainable Entrepreneurial Culture Programs Promoting Social Responsibility: A European Regional Experience. Sustainability, 11(13), 1-19, e-ISSN: 2071-1050. Recovered from:

https://doi.org/10.3390/su11133625

Torres-Porras, J., \& Arrebola, J. (2018). Construyendo la ciudad sostenible en el Grado de Educación Primaria. Revista Eureka sobre Enseñanza y Divulgación de las Ciencias, 15(2), 2501, e-ISSN: 1697-011X. Recuperado de:

https://doi.org/10.25267/Rev Eureka ensen divulg cienc.2018.v15.i2. $\underline{2501}$

Zhai, Y., Sun, W., Tsai, S., Wang, Z., Zhao, Y., \& Chen, Q. (2018). An

Empirical Study on Entrepreneurial Orientation, Absorptive Capacity, and SMEs' Innovation Performance: A Sustainable Perspective. Sustainability, 10(2), 1-14, e-ISSN: 2071-1050. Recovered from: https://doi.org/10.3390/su10020314 


\section{Artículo Original / Original Article}

\section{Miguel Jesús López Serrano \\ e-mail: mjlopez@uco.es}

Nacido en Córdoba, España, el 28 de diciembre del año

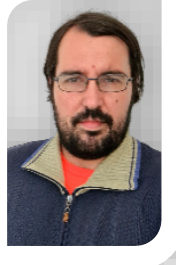
1979. Doctor en Historia Contemporánea por la Universidad de Córdoba (UCO), en España; A lo largo de estos últimos cinco años he ejercido funciones docentes en distintas universidades como la Universidad Internacional de La Rioja (UNIR), el Real Centro Universitario Escorial "María Cristina" (UCM); y desde 2018 soy profesor en el Departamento de Didácticas Específicas, de la Universidad de Córdoba; Mi trabajo se presenta como investigador en la Didáctica de la Historia, me he centrado en la enseñanza de la Historia local de Córdoba, España, las narraciones aplicadas al profesorado 
OAI-PMH: http://www.indteca.com/ojs/index.php/Revista_Scientific/oai

Artículo Original / Original Article

\section{Rafael Guerrero Elecalde}

e-mail: rgelecalde@uco.es

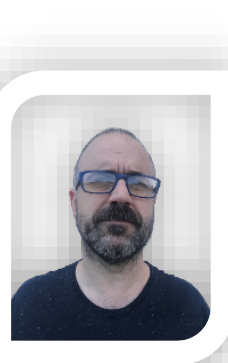

Nacido en Santoña, España, el 11 de noviembre del año 1973. Doctor en Historia en la Universidad del País Vasco (UPV/EHU), en España; A lo largo de estos últimos cinco años he ocupado el puesto de Personal Investigador Doctor Contratado (PIC), en la Universidad del País Vasco (UPV-EHU) ;y desde el año 2017, soy docente en el Departamento de Didácticas Específicas, de la Universidad de Córdoba (UCO); Mi trabajo como investigador en la Didáctica de la Historia, se ha centrado en la enseñanza de la Historia local, la historia de las Mujeres y del patrimonio, así como al uso de las fuentes documentales como recurso didáctico, entre otras.

El contenido de este manuscrito se difunde bajo una Licencia de Creative Commons ReconocimientoNoComercial-Compartirlgual 4.0 Internacional 